\title{
Adaptação transcultural e validação da Decisional Conflict Scale para utilização no Brasil
}

\author{
Adaptation and cross-cultural validation of the Decisional Conflict Scale for use in Brazil
}

Adaptación y validación transcultural de la Decisional Conflict Scale para uso en Brasil

Cintia Maria Tanure Bacelar Antunes ${ }^{1,2 *}$, Luciana Neves da Silva Bampi ${ }^{1}$, Francino Machado de Azevedo Filho ${ }^{2,3}$, Feng Yu Hua ${ }^{4}$.

\section{RESUMO}

Objetivo: Realizar a adaptação transcultural e avaliação das propriedades psicométricas da Decisional Conflict Scale para o português do Brasil. Métodos: Estudo metodológico de adaptação transcultural. Seguiu cinco etapas: tradução, síntese, tradução reversa, avaliação por comitê de especialistas e validação com 190 estudantes de enfermagem. A validade de constructo foi verificada por meio da análise fatorial exploratória, adotando-se a estatística de Kaiser-Meyer-Olkin, e as análises Screeplot e Engevalues. Utilizado o Alpha de Cronbach para avaliar a confiabilidade interna das subescalas. Resultados: O Coeficiente de Validade de Conteúdo dos itens alcançou concordância de 0,79. A estatística de Kaiser-Meyer-Olkin com resultado de 0,91 , adequados a próxima etapa. A análise Screeplot apresentou distribuição de dois fatores, confirmada pela análise Engevalues. Análise Fatorial Exploratória revelou duas subescalas: a primeira Decisão, Incerteza e Suporte e a segunda Informação e Clareza de Valores, com coeficientes de Alpha de Cronbach 0,88 e 0,89. Conclusão: A versão em português do Brasil da Decisional Conflict Scale, Escala de Conflito na Tomada de Decisão, apresentou duas subescalas com adequadas evidências de validade e confiabilidade possibilitando identificar os fatores que influenciam no conflito de decisão no processo saúde-doença.

Palavras-chave: Tomada de decisão compartilhada, Estudos de validação, Cuidado centrado no paciente.

\section{ABSTRACT}

Objective: To realize the cross-cultural adaptation and evaluation of the psychometric properties of the Decisional Conflict Scale for Brazilian Portuguese. Methods: Methodological study of cross-cultural adaptation. It followed five steps: translation, synthesis, reverse translation, evaluation by an expert committee and validation with 190 nursing students. The construct validity was verified through exploratory factor analysis, adopting the Kaiser-Meyer-Olkin statistic, and the Screeplot and Engevalues analyses. Cronbach's Alpha was used to assess the internal reliability of the subscales. Results: The Content Validity Coefficient of the items reached an agreement of 0.79 . Kaiser-Meyer-Olkin stat with a score of 0.91 , suitable for the next step. The Screeplot analysis showed a two-factor distribution, confirmed by the Engevalues analysis. The Exploratory Factor Analysis revealed two subscales: the first Decision, Uncertainty and Support and the second Information and Clarity of Values, with Cronbach's alpha coefficients of 0.88 and 0.89 . Conclusion: The Brazilian Portuguese version of the Decision Conflict Scale, Conflict in Decision Making Scale, presented two subscales with adequate evidence of validity and reliability, enabling the identification of factors that influence decision conflict in the health-disease process.

Key words: Shared decision making, Validation study, Patient-centered care.

\footnotetext{
1 Universidade de Brasília (UNB), Brasília - DF. *E-mail: cintiatanure@gmail.com.

${ }^{2}$ Secretaria de Estado da Saúde do Distrito Federal, Brasília - DF.

3 Universidade Estadual do Goiás (UEG), Formosa - GO.

${ }^{4}$ Universidade Federal do ABC, São Bernardo do Campo - SP.
} 


\section{RESUMEN}

Objetivo: Realizar la adaptación transcultural y evaluación de las propiedades psicométricas de la Escala de Conflicto Decisional al portugués brasileño. Métodos: Estudio metodológico de adaptación transcultural. Siguió cinco pasos: traducción, síntesis, traducción inversa, evaluación por un comité de expertos y validación con 190 estudiantes de enfermería. La validez de constructo se verificó mediante análisis factorial exploratorio, adoptando la estadística de Kaiser-Meyer-Olkin y los análisis Screeplot y Engevalues. Se utilizó el Alfa de Cronbach para evaluar la confiabilidad interna de las subescalas. Resultados: El Coeficiente de Validez de Contenido de los ítems alcanzó un acuerdo de 0,79. Kaiser-Meyer-Olkin con un resultado de 0,91, adecuado para el siguiente paso. El análisis Screeplot mostró una distribución de dos factores, confirmada por el análisis Engevalues. El Análisis Factorial Exploratorio reveló dos subescalas: la primera Decisión, Incertidumbre y Soporte y la segunda Información y Claridad de Valores, con coeficientes Alpha de Cronbach de 0.88 y 0.89. Conclusão: A versão em português do Brasil da Decisional Conflict Scale, Escala de Conflito na Tomada de Decisão, apresentou duas subescalas com adequadas evidências de validade e confiabilidade possibilitando identificar os fatores que influenciam no conflito de decisão no processo saúde-doença.

Palabras clave: Toma de decisiones conjunta, Estudio de validación, Atención dirigida al paciente.

\section{INTRODUÇÃO}

No decorrer da vida as pessoas necessitam tomar decisões sobre a realização de tratamentos e/ou exames diagnósticos em busca do bem-estar e da melhora de uma condição de saúde. O reconhecimento do imperativo da participação do paciente no processo decisório relacionado à sua saúde no Brasil é uma realidade recente. $O$ País tem uma tradição paternalista, na qual os profissionais de saúde determinam ao paciente o que fazer frente a uma doença ou agravo à saúde, sem considerar a opinião, os valores ou suas preferências do paciente no processo decisório (ALBUQUERQUE A e ANTUNES CMTB, 2021).

Em oposição, a Tomada de Decisão Compartilhada (TDC) é referenciada mundialmente como modelo a ser empregado na relação clínica, profissional de saúde e o paciente. Neste referencial, o envolvimento do paciente é visto como uma prerrogativa obrigatória, sendo reconhecido o direito a informações sobre sua saúde, legitimando a participação ativa e, portanto, a autonomia pessoal do paciente (LÉGARÉ F, 2018). Na TDC, para adequada decisão, a provisão de informações confiáveis é o primeiro e essencial passo (DURAND MA, et al., 2021).

A falta de informação sobre as alternativas terapêuticas, o desconhecimento sobre os riscos e os benefícios, o não esclarecimento acerca dos valores pessoais envolvidos e a falta de apoio ou a coerção durante o processo de tomada de decisão são fatores que geram incertezas. Esses elementos cognitivos, af etivos e sociais influenciam a escolha gerando conflitos de decisão (O'CONNOR AM, 1995; STACEY D, 2017).

O conflito de decisão é definido como um estado de incerteza acerca de uma ação (O'CONNOR AM, 1995). O nível de insegurança aumenta proporcionalmente as decisões que envolvem maiores riscos ou dúvidas sobre os resultados, ou aquelas que implicam possibilidade de perdas ou ganhos que são valorados de maneiras diferentes pelas pessoas (O'CONNOR AM, 1995; O'CONNOR AM, 2010).

Nesse âmbito, a Decisional Conflict Scale (DCS) é uma escala desenvolvida por Annette O'Connor, em 1995, para mensurar a percepção individual de incerteza, durante um processo de tomada de decisão em saúde. A escala também identifica os fatores contribuintes para a incerteza e a qualidade da decisão tomada (O'CONNOR AM, 1995; GARVELINK MM, 2019a). A DCS já foi traduzida para várias línguas em 20 países diferentes, tendo um incremento na utilização ao longo dos anos, passando de 18 aplicações no final dos anos 1990 para 182 intervenções nos últimos cinco anos (GARVELINK MM, 2019b).

A DCS é uma ferramenta de autoaplicação, composta de 16 itens, que utiliza o formato de resposta tipo Likert, variando entre 0 (concordo completamente), 1 (concordo), 2 (nem concordo nem discordo), 3 (discordo) e 4 (discordo fortemente). A pontuação total pode variar de 0 a 100 . Quanto maior o valor, mais presente éo conflito na tomada de decisão. As respostas aos itens são somadas e posteriormente o valor é dividido por 16 e em seguida multiplicado por 25. Resultados $\geq 37,5$ estão associados a indecisão (O'CONNOR AM, 2010). 
A DCS já foi validada no Japão (KAWAGUCHI T, et al., 2013), Coreia (KIM JS, et al., 2017), França (MARCIN J, et al., 2006), Chile (URRUTIA M, et al., 2008), Alemanha (BUCHHOLZ A, et al., 2011), Holanda (KOEDOOT N, et al., 2001), Portugal (MARTINHO MJCM, et al., 2013), além do país de origem Canadá onde é utilizada em inglês, assim como nos Estados Unidos da América (O'CONNOR AM, 1995). A validação em Portugal, utilizando o português daquele país, enfatizou, na conclusão do trabalho, a necessidade da realização de adaptação transcultural e a validação do instrumento antes da utilização em outros países de língua portuguesa, dentre eles, o Brasil (MARTINHO MJCM, et al., 2013). Após a adaptação e a validação, as versões finais são disponibilizadas no site do Hospital de Ottawa para livre utilização.

A ferramenta é constantemente utilizada com a finalidade de mensurar a ef etividade do aconselhamento feito aos pacientes no processo de TDC, sendo aplicada após decisões sobre tratamentos de saúde, exames para diagnósticos e/ou planos de cuidados em saúde (GARVELINK MM, 2019). É utilizada em diferentes contextos como na avaliação da eficácia de ajuda decisional entre homens com câncer de próstata (BERRY DL, 2018), na avaliação da efetividade do atendimento baseado em TDC versus atendimento usual em problemas relacionados à anticoagulação (KUNNEMAN M, et al., 2021), e na identificação da prevalência de conflito decisional significativo após consulta em pessoas com Diabetes Mellitus na atenção primária (BRAVO $\mathrm{P}, 2018)$.

No Brasil não há instrumento que avalie a certeza ou a indecisão do paciente após adotar uma conduta terapêutica indicada durante um encontro clinico, relação clínica. O objetivo deste estudo foi realizar a adaptação transcultural e a validação, com avaliações psicométricas, da versão adaptada da DCS para o português do Brasil mensurando a confiabilidade por meio da consistência interna.

\section{MÉTODOS}

Este estudo é o resultado de uma das etapas da pesquisa de mestrado de uma das autoras, disponível em repositório da instituição, concluído ano de 2018 (ANTUNES CMTB, 2018). Para tanto, necessitou realizar a adaptação transcultural da DCS e validação do instrumento, visto que as validações previamente citadas demonstram a importância desse procedimento para a sua utilização em cada país.

Realizou-se um estudo do tipo metodológico de adaptação cultural ajustado ao modelo proposto por Pasquali (PASQUALI L, 2010; BEATON DE, et al., 2000) e posteriormente realizou-se a validação com avaliação das propriedades psicométricas. O primeiro passo foi a obtenção de autorização da autora, Annette O'Connor, que consentiu com a realização da pesquisa, pois facilitará a utilização do instrumento para pacientes no Brasil.

Em seguida a adaptação transcultural foi realizada considerando as etapas: tradução, síntese, retro tradução e análise por juízes (PASQUALI L, 2010). Optou-se pela direta aplicação no público-alvo, confome observado em outras validações da DCS (MARTINHO MJCM, et al., 2013; URRUTIA M, et al., 2008).

A primeira etapa de tradução foi realizada partindo do instrumento original em inglês, disponível no site do Hospital de Ottawa, por dois tradutores bilíngues denominados T1 e T2. Ambos eram brasileiros e fluentes nos dois idiomas, inglês e português, e realizaram isoladamente esta etapa (PASQUALI L, 2010). Assim minimizou-se vieses linguísticos, culturais, psicológicos e de compreensão teórica e prática. Um dos tradutores era profissional de saúde e tinha conhecimento sobre os objetivos do instrumento, o outro desconhecia o tema central (PASQUALI L, 2010; FERREIRA L, et al., 2014).

A segunda etapa denominada síntese, foi realizada por uma comissão formada pelos tradutores (T1 e T2) e duas pesquisadoras do projeto. O objetivo foi comparar as distintas traduções e unificar as diferenças idiomáticas, gramaticais, conceituais e linguísticas numa versão denominada Síntese (S1) (PASQUALI L, 2010; FERREIRA L, et al., 2014).

Posteriormente, realizou-se a retrotradução, tradução reversa, na qual a versão $\mathrm{S} 1$ foi transladada para o idioma original do instrumento. Esta etapa foi realizada por dois retro-tradutores (RT1 e RT2) de forma independente, sem conhecimento do instrumento original. Foram convidados dois nativos de língua inglesa, com domínio do português do Brasil, que residiram ou residem no País, devido ao conhecimento e vivência 
com a língua e a cultura brasileira. O objetivo foi identificar se a versão traduzida (S1) refletia os conteúdos do instrumento original confirmando a consistência da tradução, dando origem assim a versão Preliminar $(P)$ (FERREIRA L, et al., 2014).

A versão $P$ passou pela validação de conteúdo por juízes, que avaliaram todos os itens do instrumento quanto a clareza de linguagem, a pertinência prática e a relevância teórica (PASQUALI L, 2010). Na formação do comitê de juízes adotou-se a composição de: um profissional de saúde, um metodologista na temática de validação de instrumentos e especialista na área de ética e bioética, uma enfermeira gestora de saúde de um grande centro de atendimento público, um linguista e um usuário dos serviços de saúde conforme referência da temática. (FERREIRA L, et al., 2014).

Todos receberam o convite, o termo de consentimento livre e esclarecido (TCLE) e as orientações para participar da pesquisa por meio da plataforma surveymonkey ${ }^{\circledR}$. Após aceitarem participar foram direcionados para avaliação dos itens dentro dos critérios descritos, a pontuação foi disponibilizada em escala tipo Likert. Os dados resultantes dessa avaliação foram analisados por meio do programa Statistical Package for the Social Sciences (SPSS) versão 24. Foi realizado cálculo de Coeficiente de Validade de Conteúdo (CVC), conforme descrito por Hernández-Nieto, na análise das respostas dos juízes (PASQUALI L, 2010). Essa avaliação originou a versão adaptada da DCS para o português do Brasil, que passou a ser denominada Escala de Conflito na Tomada de Decisão (ECTD).

Na verificação da validade de constructo da ECTD foi realizada aplicação em estud antes de enfermagem que aceitaram participar dessa etapa, sendo do primeiro ao quarto ano, amostra não-aleatória, seguindo o mesmo método utilizado no processo de validação da escala original, assim como na versão do Chile e de Portugal (O'CONNOR AM, 1995; URRUTIA M, et al., 2008; MARTINHO MJCM, et al., 2013). Os estudantes não tinham conhecimento prévio deste instrumento. Para o cálculo da amostra, a literatura recomenda dez participantes por item do instrumento a ser avaliado (PASQUALI L, 2010). Como a ferramenta é composta por 16 itens, deveriam ser arrolados na pesquisa 160 participantes, contudo foram abordados 190 est udantes com intuito de ter uma margem de segurança, para itens sem resposta ou questionários em branco.

Os dados obtidos foram analisados por meio foi de análise fatorial exploratória, através do SPSS24, por se tratar de escala multidimensional. Com intenção de verificar a conformidade dos dados para a análise fatorial foi utilizado o índice de Kaiser-Meyer-Okin (KMO) (FIGUEIREDO FILHO DB, et a. 2014). A consistência interna foi verificada por meio do coeficiente Alfa de Cronbach $(\alpha)$, recomendado neste tipo de estudo (ECHEVARRÍA-GUANILO ME, et al., 2017). O Alfa de Cronbach $\alpha>0,8$ revela consistência muito boa (FIGUEIDERO FILHO DB, et al., 2014). Utilizou-se a análise gráfica de Scree plot, análise da variância acumulada e a técnica de Kaiser (eigenvalue) para extração de fatores (FIGUEIDERO FILHO DB, et al., 2014).

O estudo foi aprovado pelo Comitê de Ética em Pesquisa (CEP) da Fundação de Ensino e Pesquisa em Ciências da Saúde da Secretaria de Estado de Saúde do Distrito Federal, tendo CAAE: 67372017.6.0000.5553, protocolo № 2.080.560. Foi assegurado aos participantes, a confidencialidade e a privacidade de acordo com a Resolução 466/2012 do Conselho Nacional de Saúde (CNS).

\section{RESULTADOS}

O processo de tradução e de síntese do instrumento original, foi realizado sem dificuldades por se tratar de um instrumento com linguagem simples e apenas 16 itens (Quadro 1). Como descrito na literatura um dos tradutores apresentou uma linguagem mais próxima da população geral e o outro um vocabulário mais técnico por ser profissional da área da saúde (FERREIRA L, et al., 2014). A versão Síntese (S) foi finalizada após reunião entre os tradutores e os pesquisadores, com discussão detalhada de cada item do instrumento, averiguando as diferenças de expressão buscando avaliar a equivalência semântica entre o instrumento original e o traduzido, além da correspondência conceitual e experiencial.

$\mathrm{Na}$ retrotradução realizada por duas pessoas independentes, as versões resultantes foram comparadas com o instrumento original e verificou-se equivalência em vários itens, assegurando a consistência da tradução e conceitual. Demonstrou-se que a versão síntese, então denominada Preliminar (P), era adequada para a avaliação seguinte. 
Na avaliação dos cinco juízes primeiramente foi examinada a validade semântica da versão $P$, na qual todos os itens alcançaram $80 \%$ de concordância, valor considerado satisfatório, demonstrando serem compreensíveis para aplicação na população brasileira (PASQUALI L, 2010). Os testes do Coeficiente de Validade de Conteúdo (CVC) apresentaram no quesito clareza o valor de 0,78 , pertinência prática 0,79 e relevância teórica 0,80 , com um CVC total de 0,79 .

Aceitaram participar da validação da ECTD 190 discentes de enfermagem. A amostra de conveniência foi composta por maioria de mulheres (80\%), com idade entre 17 e 44 anos. A idade média foi 21,85 e o desvio padrão 4,72 anos. A distribuição percentual dos participantes do estudo de acordo com o ano do curso foi de $24,7 \%$ na $1^{a}, 23,7 \%$ na $2^{a}, 23,7 \%$ na $3^{\text {a }}$ e $27,9 \%$ na $4^{\underline{a}}$ série.

A questão envolvida na validação da ECTD dizia respeito ao processo decisório frente ao estado gripal. Dos participantes, $91 \%$ referiram já haver realizado algum tipo de tratamento e 6,3\% relataram já ter estado hospitalizado devido à gripe. Diante das escolhas frente ao estado gripal a maioria dos participantes, $65 \%$, afirmou fazer uso de medicamentos para melhoria dos sintomas, seguida pela vacinação anual, $18,4 \%$. Obtiveram menores índices aguardar a resolução natural da doença e medidas de higiene pessoal e distanciamento social com $9,5 \%$ e $6,8 \%$ respectivamente.

Para verificar a possibilidade de análise fatorial dos dados obtidos por meio da coleta com os envolvidos na validação, primeiramente calculou-se a estatística de ajuste de Kaiser-Meyer-Olkin (KMO). Esse cálculo demonstrou valor de 0,91 , sendo adequado pois quanto mais perto de um, mais adequada é a aplicação da análise fatorial exploratória (PASQUALI L, 2010; FIGUEIDERO FILHO DB, et al., 2014). O número mínimo de respostas válidas considerado foi dez vezes o número de itens do instrumento (PASQUALI L, 2010; FERREIRA L, et al., 2014; FIGUEIDERO FILHO DB, et al., 2014).

A análise Screeplot (Diagrama de Sedimentação), auxilia no processo de agrupamento dos itens daescala em fatores. No presente estudo esta análise apontou a divisão dos itens do instrumento em 2 fatores. No eixo das abcissas, que corresponde aos números de fatores, pode-se observar que no número 2 há uma quebra no traçado, modificando o trajeto da curva. Portanto evidencia a presença de dois fatores a serem extraídos, pois após o número 2 há uma horizontalização dos resultados (Figura 1).

Figura 1 - Diagrama de segmentação da análise dos dados coletados com os estudantes de enfermagem.

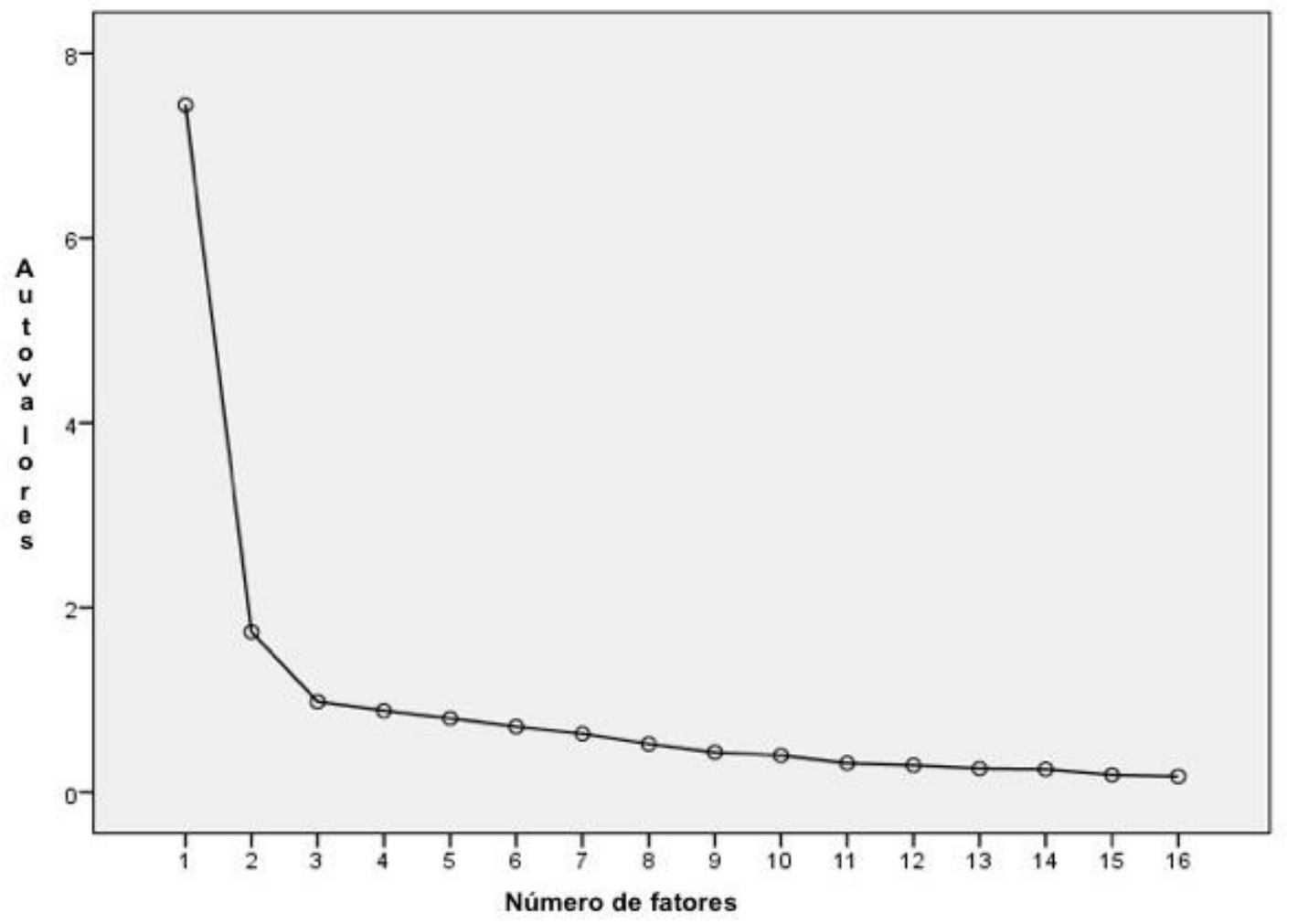

Fonte: Antunes CMTB, et al., 2021. 
O mesmo resultado de extração foi obtido na análise dos Engevalues (Autovalores) com valor acima de 1: Fator $1=7,441$ e Fator $2=1,736$. Na variância acumulada esses fatores somados explicaram $57,35 \%$ do total de variância do instrumento (Tabela 1).

Tabela 1 - Percentual da variância total entre os fatores da Escala de Conflito na Tomada de Decisão.

\begin{tabular}{cccc}
\hline \multirow{2}{*}{ Fator } & \multicolumn{3}{c}{ Valores Iniciais } \\
\cline { 2 - 4 } & Total & Percentual da Variância & Percentual Cumulativo \\
\hline 1 & 7,441 & 46,506 & 46,506 \\
2 & 1,736 & 10,847 & 57,353 \\
3 &, 980 & 6,124 & 63,477 \\
4 &, 883 & 5,521 & 68,998 \\
5 &, 799 & 4,995 & 73,993 \\
6 &, 709 & 4,429 & 78,422 \\
7 &, 633 & 3,956 & 82,377 \\
8 &, 523 & 3,268 & 85,645 \\
9 &, 432 & 2,700 & 88,345 \\
10 &, 398 & 2,487 & 90,832 \\
11 &, 312 & 1,952 & 92,784 \\
12 &, 292 & 1,824 & 94,608 \\
13 &, 255 & 1,596 & 96,203 \\
14 &, 248 & 1,551 & 97,754 \\
15 &, 188 & 1,175 & 98,929 \\
16 &, 171 & 1,071 & 100,000 \\
\hline
\end{tabular}

Nota: Método de extração: Análise Fatorial Exploratória; Método de rotação: Varimax.

Fonte: Antunes CMTB, et al., 2021.

A extração utilizada na Análise Fatorial Exploratória considerou que os itens fariam parte de um determinado fator (divisão interna da escala) se apresentasse um valor de carga superior a 0,4 . Todos os itens foram incluídos com distribuição resultante em dois fatores. Os itens que na escala original compunham a subescala denominada incerteza (itens 10, 11 e 12) e decisão ef etiva (itens 13, 14, 15 e 16), ficaram alocados no fator 1 juntamente com os itens que na escala original eram da subescala denominada suporte a decisão (itens $7,8,9$ ). Os demais itens que na escala original compunham a dimensão de fatores que se relacionavam a subescala denominada informação adequada (itens $1,2,3$ ) e respeito a clareza de valores (itens $4,5,6$ ) ficaram no fator 2 (Tabela 2).

Tabela 2 - Resultado da extração de fatores na aplicação dos estudantes de enf ermagem.

\begin{tabular}{cccc}
\hline & & & Fator \\
\cline { 2 - 3 } Itens & $\mathbf{1}$ & $\mathbf{2}$ \\
\hline Item 1 & - &, 656 \\
Item 2 & - &, 771 \\
Item 3 & - &, 694 \\
Item 4 & - &, 828 \\
Item 5 & - &, 728 \\
Item 6 & - & - \\
Item 7 &, 548 & - \\
Item 8 &, 432 & - \\
Item 9 &, 581 & - \\
Item 10 &, 714 & - \\
Item 11 &, 744 & - \\
Item 12 &, 624 & - \\
Item 13 &, 690 & - \\
Item 14 &, 645 & - \\
Item 15 &, 670 & - \\
Item 16 &, 674 & \\
\hline
\end{tabular}

Nota: Método de Extração: Análise Fatorial Exploratória; Método de Rotação: Varimax

Fonte: Antunes CMTB, et al., 2021. 
A consistência interna dos fatores foi calculada através do Alpha de Cronbach apresentando para o fator 1 valor de 0,88 e para o fator 2 o valor de 0.89 . O título designado para a versão brasileira da DCS validada no presente estudo foi Escala de Conflito na Tomada de Decisão (ECTD) (Quadro 1).

Quadro 1 - Escala de Conflito na Tomada de Decisão (ECTD) - versão para uso no Brasil da Decisional Conflict Scale.

\begin{tabular}{|c|c|c|}
\hline Subescala & Itens da escala & Afirmações \\
\hline \multirow{6}{*}{$\begin{array}{l}\text { Informação e Clareza } \\
\text { de Valores }\end{array}$} & Item 1 & Eu sei quais opções estão disponíveis para mim. \\
\hline & Item 2 & Eu conheço os benefícios de cada opção. \\
\hline & Item 3 & Eu conheço os riscos e os ef eitos colaterais de cada opção. \\
\hline & Item 4 & Eu compreendo quais benefícios mais importam para mim. \\
\hline & Item 5 & $\begin{array}{l}\text { Eu compreendo quais riscos e quais ef eitos colaterais mais } \\
\text { importam. }\end{array}$ \\
\hline & Item 6 & $\begin{array}{l}\text { Eu compreendo sobre o que é mais importante para mim (os } \\
\text { benefícios ou os riscos e os ef eitos colaterais). }\end{array}$ \\
\hline \multirow{10}{*}{$\begin{array}{l}\text { Decisão, Incerteza e } \\
\text { Suporte }\end{array}$} & Item 7 & $\begin{array}{l}\text { Eu tenho apoio suficiente dos outros para realizar uma } \\
\text { decisão. }\end{array}$ \\
\hline & Item 8 & Eu estou decidindo sem a pressão de outras pessoas. \\
\hline & Item 9 & Eu tenho orientação o suficiente para realizar uma escolha. \\
\hline & Item 10 & Eu estou certo sobre qual a melhor escolha para mim. \\
\hline & Item 11 & Eu me sinto seguro sobre o que escolher. \\
\hline & Item 12 & Essa decisão é fácil para eu tomar. \\
\hline & Item 13 & Eu sinto que fiz uma escolha orientada e consciente. \\
\hline & Item 14 & Minha decisão demonstra o que é importante para mim. \\
\hline & Item 15 & Eu espero permanecer com a minha decisão. \\
\hline & Item 16 & Eu estou satisf eito com a minha decisão. \\
\hline
\end{tabular}

Fonte: Antunes CMTB, et al., 2021.

Quanto ao conflito de decisão entre os participantes da validação, identificou-se um percentual total de $20,53 \%$, sendo considerados aqueles que obtiveram pontuação $\geq 37,5$. Os acadêmicos da $2^{a}$ série apresentaram maior grau de conflito frente a tomada de decisão (Tabela 3 ).

Tabela 3 - Distribuição de estudantes por série de acordo com pontuação na Escala de Conflito na Tomada de Decisão (ECTD) Brasília, 2018. ( $n=190)$.

\begin{tabular}{ccc}
\hline Nível & Percentual de decisão efetiva & Percentual de conflito de decisão \\
\hline $1^{\underline{a}}$ série & $70,21 \%$ & $29,79 \%$ \\
$2^{\underline{a}}$ série & $64,44 \%$ & $35,56 \%$ \\
$3^{\underline{a}}$ série & $84,44 \%$ & $15,56 \%$ \\
$4^{\underline{a}}$ série & $96,33 \%$ & $3,77 \%$ \\
\hline
\end{tabular}

Fonte: Antunes CMTB, et al., 2021.

\section{DISCUSSÃO}

A participação do paciente no processo de tomada de decisão em saúde é tônica do momento no que se refere a relação clínica. Um número crescente de ferramentas de avaliação está disponível para apoiar essa participação em vários países, porém, no Brasil ainda são incipientes as discussões e a utilização de instrumentos nessa área. Esta lacuna foi o principal motivador para buscar uma ferramenta que avaliasse as várias dimensões influenciadoras da tomada de decisão em saúde, incentivando a abordagem do tema e a participação dos usuários. A justificativa para a eleição da DCS ocorreu por se tratar de uma escala validada e utilizada em ampla gama de idiomas e contextos (KAWAGUCHI T, et al., 2013; MARTINHO MJCM, et al., 2013). 
Na etapa de avaliação dos juízes, os testes de coeficiente de validade de conteúdo (CVC) foram considerados satisfatórios com valor total de 0,79 , não sendo necessárias adaptações no conteúdo da versão do instrumento analisada nessa fase (ALEXANDRE NMC, et al., 2011). Esta etapa contribuiu de forma significativa pois abrangeu diferentes profissionais com experiências e pensamentos diversos sobre o tema que avaliaram positivamente a compreensão e a coerência cultural além da equivalência de conteúdo.

O alcance de 190 participantes, no processo de validação, possibilitou a avaliação das propriedades psicométricas da ECTD, versão da DCS para utilização no Brasil. O predomínio de mulheres (80\%), dentre os estudantes de enfermagem participantes, corroborou com a última caracterização da profissão no País realizada pelo Conselho Federal de Enfermagem (COFEN) (MACHADO MH, et al., 2015).

A análise fatorial é a redução de dados, de forma que um grande número de variáveis possa ser reduzido a um conjunto menor de índices que tenha uma máxima variabilidade e fidedignidade (ALEXANDRE NMC, COLUCl MZO, et al., 2011). A alteração da quantidade de fatores encontrados na presente análise, que resultou em dois fatores com variância total de $57,35 \%$, não acarretou prejuízos para a validação do instrumento. Fato semelhante ocorreu na validação da DCS em outros países como Chile, França e Holanda, para os quais resultaram 4 fatores, Japão e Alemanha, nos quais 5 fatores foram obtidos, e Coréia, para a qual, como ocorreu na presente validação, 2 fato res foram alcançados (KAWAGUCHI T, et al., 2013; KIM JS, et al., 2017; MANCIN J, et al., 2006; URRUTIA M, et al., 2008; BUCHHOLZ A, et al., 2011; KOEDOOT N, et al., 2001).

A análise da consistência interna nas subescalas variando entre 0,88 e 0,89 , apresentou resultado similar comparado aos encontrados na validação original que variou entre 0,78 a 0,92 e na versão portuguesa que variou entre 0,73 e 0,93 (O'CONNOR AM, 1995; MARTINHO MJCM, et al., 2013).

Como ocorrido na tradução e na validação da DCS em outras línguas, esse processo buscou ajustar a nomenclatura utilizada para as subescalas das demais versões (O'CONNOR AM, 1995; URRUTIA M, et al., 2008). Houve a necessidade de algumas mudanças na alocação dos itens que compõem as subescalas. $O$ fator 1 constituiu-se de itens da escala original relacionados a incerteza, ao suporte de decisão e a decisão ef etiva, sendo denominado na atual validação de Decisão, Incerteza e Suporte. $O$ fator 2 , relacionava-se a informação adequada (sobre opções de tratamento, riscos e benefícios) e a clareza de valores pessoais, agora chamado Informação e Clareza de Valores. O título designado para o instrumento validado no presente estudo, Escala de Conflito na Tomada de Decisão, foi considerado mais próximo ao título original.

A avaliação quanto ao conflito de decisão dos estudantes de enfermagem frente a síndrome gripal não foi o objeto principal desta pesquisa, constituiu o processo de validação, contudo, observou-se que o uso de medicamentos para melhoria dos sintomas apresentou-se como a opção mais frequentemente adotada (65\%) pelos participantes. Esse resultado corrobora outro estudo que reflete a baixa cobertura vacinal em estudantes de enfermagem (MARQUES ADB, et al., 2013).

O conflito de decisão relacionada a síndrome gripal ocorreu principalmente entre estudantes da segunda série $(35,56 \%)$, com menor índice foi encontrado entre alunos da quarta série (3,77\%). Essa importante variação percentual pode estar relacionada a aquisição de conhecimentos sobre a patolog ia, a clínica e as intervenções na prevenção e no tratamento da doença fazendo com que os discentes da quarta série se sentissem mais seguros quanto as decisões a serem tomadas. Estudos sobre a tomada de decisão indicam que a falta de informações relevantes dificulta a tomada de decisão tornando as pessoas menos propensas a realizar uma escolha harmônica com seus valores e objetivos (STACEY D, et al., 2015).

Considera-se uma limitação deste estudo a utilização de apenas uma amostra populacional para a validação da ECDT. Recomenda-se a aplicação em outros contextos no Brasil para consolidar a utilização do instrumento na avaliação do processo de tomada de decisão. Como se trata da primeira aplicação da ferramenta no País, novos estudos são necessários para a confirmação os resultados obtidos e o aprofundamento da aplicabilidade desse instrumento na população brasileira. Como o Brasil é um país de dimensões continentais podem existir diferenças culturais, educacionais e regionais que influenciem na aplicação da escala. 


\section{CONCLUSÃO}

Os resultados psicométricos da aplicação da Escala de Conflito na Tomada de Decisão, versão adaptada da Decisional Conflict Scale para o português do Brasil, encontrou índices de consistência interna adequados para avaliação de conflitos de decisão na área da saúde. A validação dessa escala possibilita identificar as situações análogas entre diferentes nações, dentro do processo de tomada de decisão, principalmente nas questões de saúde, assunto já em ampla discussão mundial. A utilização desse instrumento poderá contribuir para o conhecimento relacionado a temática assistência/cuidado centrado no paciente, auxiliando na conscientização de usuários e de profissionais da saúde sobre os direitos do paciente e sua participação na tomada de decisão em relação ao corpo e a saúde.

\section{AGRADECIMENTOS}

Os autores agradecem a professora Annette O'Connor que gentilmente autorizou a utilização da DCS para este estudo. Aos tradutores Filipe Wierdehecker e Ariel Oliveira, assim como aos retro-tradutores Samantha Jo Monnahan e Geoff Dyer. Agradecimentos a professora Luciana Melo que possibilitou a abordagem dos estudantes de enfermagem, extensivos a Escola Superior Ciências da Saúde - DF (ESCS), aos 190 alunos e aos 5 juízes de construto que aceitaram participar do processo de adaptação e de validação.

\section{REFERÊNCIAS}

1. ALBUQUERQUE A, ANTUNES CMTB. To mada de decisão compartilhada na saúde: ap roximações e distanciamentos entre a ajuda decisional e os apoios de to mada de decisão. Cad. Ibero-amer. Dir. Sanit., 2021; 10(1): 203-223.

2. ALEXANDRE NMC, COLUCI MZO. Content validity in the development and adaptation process of measurement instruments. Cien Saude Colet., 2011; 16: 3061-8.

3. ANTUNES CMTB. Tradução, validação e aplicação da Decisional Conflict Scale no Brasil. Dissertação (Mestrado em Enfermagem) - Programa de Pós-Graduação em Ẻnfermagem. Universidade de Brasilia, Brasilia, 2018; 114 p.

4. BEATON DE, et al. Cross-cultural adaptation of self-report measures. Spine, 2000;25(24):3186-3191.

5. BERRY DL, et al. Decision support with the personal patient profile prostate: a multicenter randomized trial. The Journal of urology, 2018; 1999(1): 89-97.

6. BRAVO P, et al. Conflicto decisional en personas con diabetes mellitus e hipertensión arterial usuarias del nivel primario de atención de salud de Chile. Rev Med Chile, 2018;146: 1286-1293.

7. BUCHHOLZ A, et al. German-language version of Decisional Conflict Scale. Zeitschriftfür klinische Psychologie und Psychotherapie, 2011; 4: 15-30

8. DURAND MA, et al. Can shared decision making address COVID-19 vaccine hesitancy? BJM Evidence-Based Medicine, $2021 ; 1-3$.

9. ECHEVARRÍA-GUANILO ME, et al. Psychometric properties of mensurement instruments: conceptual bases and evaluation methodspart I. Texto Contexto Enferm, 2017;26(4): e1600017.

10. FERREIRA L, et al. AAOS / IWH guide: Suggestions for cross-cultural scale adaptation. Avaliação Psicológica, 2014; 13(21): 45761.

11. FIGUEIREDO FILHO DB, et al. Análise fatorial garantida ou seu dinheiro de volta: uma introdução a redução de dados. Revista Eletrônicade Ciência Politica, 2014;5(2).

12. GARVELINK MM, et al. Decisional Conflict Scale use over 20 years: the anniversary review. Medical Decision Making, 2019; 39(4): 301-314.

13. GARVELINK MM, et al. Decisional Conflict Scale findings among patients and surrogates making health decisions: part II of an anniverșary review. Medical Decision Making, 2019; 39(4): 315-326.

14. LÉGARÉF et al. Interventions forincreasing the use of shared decision making by healthcare professionals. Cochrane Database of Systematic Reviews, 2018; 7.

15. MACHADO MH, et al. Características Gerais da Enfermagem: o perfil sócio demográfico. Enferm Foco, 2015;6(1/4):11-17.

16. MANCIN J, et al. Cross-cultural validation of the Decisional Conflict Scale in a sample of French patients. Quality of life research, 2006; 15: 1063-1068.

17. MARTINHO MJCM. Scale of Conflict in health care decision-making: an instrument adapted and validated for the Portuguese language. Rev Esc Enferm USP, 2013; 47(3): 576-583.

18. KAWAGUCHI T, et al. Development and validation of the Japanese version of the Decisional Conflict Scale to investigate the value of pharmacists' information: a before and after study. Medical Informatics Decis Making, 2013; 13(50): 1-8.

19. KIM JS, et al., Validation of the decisional Conflict Scale for evaluating advance care decision conflict in community-dwelling older adults. Asian Nursing Research, 2017; 11(4): 297-303.

20. KOEDOOT N, et al. The decisional conflict scale: furthervalidationin two samples of dutch oncology patients. Patient Education and Counseling, 2001; 45:187-193.

21. KUNNEMAN M, et al. Co-creating sensible care plans using decision making: patient's reflections and observations of encounters. Patiente Education and Counseling, 2021.

22. O'CONNOR AM. Validation of a decisional conflict scale. Med Decis Making, 1995; 15(1): 25-30

23. O'CONNOR AM. User Manual - Decicion Conflict Scale.

24. PASQUALI L. Instrumentação psicológica:fundamentos e praticas. Porto Alegre: Artmed, 2010.

25. STACEY D, et al. Engag ing patients using an interprofessional approach to shared decision Making. Can Oncol Nurs J., 2015; 25(4): 455-69.

26. STACEY D, et al. Decision aids for people facing health treatment or screening decisions. Cochrane Database of Systematic Reviews, 2017; 4.

27. URRUTIAM, et al. Validation of a Span ish version of the Decisional Conflict Scale. Ver Med Chile, 2008; 136: 1439-1447. 